\title{
Protective effect of resveratrol on spermatozoa function in male infertility induced by excess weight and obesity
}

\author{
XIANGRONG CUI $^{1}, \mathrm{XUAN} \mathrm{JING}^{2}, \mathrm{XUEQING} \mathrm{WU}^{1}$ and MEIQIN YAN ${ }^{1}$ \\ ${ }^{1}$ Reproductive Medicine Center, Children's Hospital of Shanxi and Women Health Center of Shanxi, Taiyuan, \\ Shanxi 030000; ${ }^{2}$ Clinical Laboratory, Shanxi Province People's Hospital, Taiyuan, Shanxi 030000, P.R. China
}

Received October 7, 2015; Accepted September 1, 2016

DOI: $10.3892 / \mathrm{mmr} .2016 .5840$

\begin{abstract}
Male infertility is a complex, multifactorial and polygenic disease that contributes to $\sim 50 \%$ cases of infertility. Previous studies have demonstrated that excess weight and obesity factors serve an important role in the development of male infertility. An increasing number of studies have reported that resveratrol may regulate the response of cells to specific stimuli that induce cell injury, as well as decrease germ cell apoptosis in mice or rats. In the present study, the semen quality and serum sex hormone levels were evaluated in 324 men, which included 73 underweight, 82 normal weight, 95 overweight and 74 obese men. All patients were referred to The Reproductive Medicine Center of Shanxi Women and Infants Hospital (Taiyuan, China) between January 2013 and January 2015. The aim of the present study was to investigate the effects of resveratrol treatment on the motility, plasma zinc concentration and acrosin activity of sperm from obese males. The sperm concentration, normal sperm morphology, semen volumes, DNA fragmentation rates and testosterone levels in men from the overweight and obese groups were markedly decreased when compared with men in the normal weight group. In addition, the progressive motility, seminal plasma zinc concentration and spermatozoa acrosin activity were notably decreased in the obese group compared with the normal weight group. However, estradiol levels were significantly increased in the overweight, obese and underweight groups compared with the normal weight group. Notably, semen samples from obese males with astenospermia treated with $0-100 \mu \mathrm{mol} / 1$ resveratrol for $30 \mathrm{~min}$ demonstrated varying degrees of improvement in sperm motility. When these semen samples were treated with $30 \mu \mathrm{mol} / 1$ resveratrol, sperm motility improved when compared to other doses of
\end{abstract}

Correspondence to: Dr Xueqing Wu, Reproductive Medicine Center, Children's Hospital of Shanxi and Women Health Center of Shanxi, 13 Xinmin North Street, Xinghua Ling, Taiyuan, Shanxi 030000, P.R. China

E-mail: wuxueqq@hotmail.com

Key words: overweight, obesity, sperm quality, resveratrol, infertility resveratrol. Therefore, $30 \mu \mathrm{mol} / 1$ resveratrol was selected for further experiments. Upon treatment of semen samples with resveratrol $(30 \mu \mathrm{mol} / \mathrm{l})$ for $30 \mathrm{~min}$, the seminal plasma zinc concentration and spermatozoa acrosin activity increased significantly in the experimental group compared with the control group. These data suggest that male obesity negatively impacts on male reproductive potential, not only through altering hormone levels, but also by directly altering sperm function. In addition, resveratrol may have a therapeutic and protective effect against obesity-induced abnormalities in semen.

\section{Introduction}

Infertility is a devastating problem of human reproduction, and male infertility contributes to $50 \%$ of all infertility cases $(1,2)$. In recent years, an increasing number of reports have demonstrated a global trend indicating a significant deterioration in male reproductive function (3-5). In parallel, obesity rates are increasing rapidly worldwide $(6,7)$, which is not only associated with an increased risk of developing chronic diseases, but has also been demonstrated to increase the risk of developing reproductive disorders (8). Due to the reported association between obesity and reproductive disorders, a greater clinical awareness and understanding of the underlying mechanisms of the effects of obesity on fertility are urgently required in order to determine the appropriate interventions.

Studies have determined that the natural polyphenol, resveratrol, has a similar molecular structure to diethylstilbestrol and estradiol (E2), and is produced in several plants in response to injury, stress, bacterial or fungal infection, ultraviolet (UV) radiation or exposure to ozone (9-11). Resveratrol is known for its anti-inflammatory, antioxidant, analgesic, cardioprotective, anti-aging and neuroprotective roles (11-13). Studies have demonstrated that resveratrol may inhibit cell apoptosis, thereby providing protection from numerous diseases, including atherosclerosis, cerebral ischemia and myocardial ischemic reperfusion injury $(14,15)$. Juan et al $(16)$ reported that resveratrol is able to decrease germ cell apoptosis in mice and rats, and serves a protective role in the male reproductive tract, as well as enhancing blood testosterone levels, testicular sperm count and epididymis sperm motility in rabbits.

The aim of the present study was to investigate the association between obesity and a number of sperm parameters, and 
to assess the protective effects of resveratrol on preventing the harmful effects of obesity on spermatozoa. This may lead to the development of strategies that facilitate the maintenance of fertility.

\section{Materials and methods}

Study population. The study population consisted of 324 men (mean age, 37.2 \pm 0.89 years) referred to the Reproductive Medicine Center of Shanxi Women and Infants Hospital (Taiyuan, China) between January 2013 and January 2015. All subjects were of Han ethnicity and from the Shanxi Province in northern China. The present study was approved by the Reproductive Medicine Ethics Committee of Shanxi Women and Infants Hospital (Taiyuan, China) and written informed consent was obtained from all subjects and their partners prior to enrollment.

The inclusion criteria for the study were as follows: i) Subjects had to be male and part of a couple that had been unable to conceive for $>1$ year; ii) the couple had regular intercourse, and iii) they were receiving infertility treatment at the Reproductive Medicine Center, Shanxi Women and Infants Hospital over the study period. Primary infertility was diagnosed after the following medical assessments were performed: The patient's medical history was examined; a clinical examination; semen analysis; semen culture for mycoplasma ureliticum and chlamydia detection; analysis of follicle-stimulating hormone (FSH), luteinizing hormone ( $\mathrm{LH}$ ), testosterone and E2 levels; a prolactin assay; and sonography of the genitalia. Each subject underwent a careful anamnesis to exclude systemic diseases, alcohol consumption, smoking, occupational chemical exposure, a history of (or presence of) endocrine disorders, testicular diseases (for example, cryptorchidism, orchitis and varicocelle), infectious genital diseases, leukocytospermia (seminal white blood cell count $>1 \times 10^{6} / \mathrm{ml}$ ), azoospermia and treatment with drugs or the use of antioxidant supplements within 3 months prior to enrollment.

A brief medical background for each subject was collected by conducting an informal interview, using clinical notes or using a self-report questionnaire. The male subjects were divided into the following four groups according to the International Association for the Study of Obesity criteria (17): Underweight [body mass index, $(\mathrm{BMI})<18.5 \mathrm{~kg} / \mathrm{m}^{2}$ ], normal weight $\left(18.5 \leq \mathrm{BMI}<23 \mathrm{~kg} / \mathrm{m}^{2}\right)$, overweight $\left(23 \leq \mathrm{BMI}<25 \mathrm{~kg} / \mathrm{m}^{2}\right)$ and obese $\left(\mathrm{BMI} \geq 25 \mathrm{~kg} / \mathrm{m}^{2}\right)$ groups.

Serum collection and analysis. Blood samples were obtained between 8:00 and 9:00 a.m. following a 12-h overnight fast, and were immediately drawn and collected in a tube containing ethylenediamine tetra-acetic acid. Samples were separated by centrifugation at $18,00 \times \mathrm{g}$ for $15 \mathrm{~min}$ at room temperature and the serum and buffy coat were separated. The serum was stored at $-80^{\circ} \mathrm{C}$ until downstream analyses were performed.

The hormone levels, including FSH (reference value, 2.10-18.6 mIU/ml), LH (reference value, 1.7-11.2 mIU/ml), E2 (reference value, $\leq 77 \mathrm{pg} / \mathrm{ml}$ ), progesterone (reference value, $\leq 0.46 \mathrm{ng} / \mathrm{ml}$ ), testosterone (reference value, $262.0-870.0 \mathrm{ng} / \mathrm{dl}$ ) and prolactin (PRL; reference value: $2.10-18.60 \mathrm{mIU} / \mathrm{ml}$ ) were measured using an AIA-2000 ST Automated Immunoassay Analyzer (Tosoh Corporation, Tokyo, Japan).
Semen collection and analysis. Semen samples were obtained by masturbation following 2-7 days of abstinence from sexual intercourse, in order to conduct a routine sperm count according to the World Health Organization (WHO, 2010) criteria (18) for sperm concentration, motility, morphology and viability. Samples were collected in sterile containers and allowed to liquefy at $37^{\circ} \mathrm{C}$ for $20 \mathrm{~min}$. Briefly, ejaculated volumes were estimated by specimen weight, and a semen density of $1.0 \mathrm{~g} / \mathrm{ml}$ was assumed. Sperm concentration, motility and viability were assessed using a Sperm Class Analyzer CASA System (Microoptic S.L, Barcelona, Spain). Sperm motility was analyzed according to the WHO (2010) guidelines (18) for determining progressive motility, non-progressive motility and immotility. Sperm morphology was assessed using David's classification system (19).

Sperm viability analysis. Sperm viability was assessed within $30 \mathrm{~min}$ of ejaculation using eosin Y staining. This was achieved by dissolving $1 \mathrm{~g}$ eosin with $1 \mathrm{~g}$ fresh sperm. The percentage of viable sperm (unstained sperm heads) and non-viable sperm (stained sperm heads) was assessed by counting a minimum of 200 spermatozoa for each sample. Each sample was analyzed in duplicate.

UV spectrophotometric assay for spermatozoa acrosin activity. Spermatozoa samples from each group were analyzed for acrosin activity using the Human Spermatozoa Acrosin Activity Quantitative assay kit (Huakang Biotechnology Development Co., Ltd., Changsha, China) according to the manufacturer's protocol. The required amount of semen $\left(7.5 \times 10^{6}\right.$ sperm) was transferred into a plastic tube and centrifuged at 2,000 x $g$ for $20 \mathrm{~min}$ at room temperature. The supernatant was removed, and the tube was inverted on absorbent paper to remove residual seminal plasma. A total of $100 \mu \mathrm{l}$ inhibitor solution $(0.3 \mathrm{~g} / \mathrm{ml}$ HEPES) and $1 \mathrm{ml}$ reactive liquid ( $0.6 \mathrm{~g} / \mathrm{ml} \mathrm{N}$ - $\alpha$-benzoyl-L-arginine-p-nitroanilide) were added to the sample and control tubes, prior to the addition of $100 \mu \mathrm{l}$ stop solution $(8.5 \mathrm{~g} / \mathrm{ml}$ benzamidine $)$ to the control tube. Sample and control tubes were then thoroughly mixed and incubated at $24^{\circ} \mathrm{C}$ for $1 \mathrm{~h}$. Stop solution (100 $\left.\mu \mathrm{l}\right)$ was added to the control tube and mixed thoroughly, and subsequently both tubes were centrifuged at $2,000 \times \mathrm{g}$ for $10 \mathrm{~min}$ at $24^{\circ} \mathrm{C}$. At this temperature, the amount of substrate that hydrolyzes $1.0 \mu \mathrm{mol} \mathrm{N}$-benzoyl-DL-arginine-4-nitroanilide hydrochloride/min is defined as 1 IU acrosin activity. Acrosin activity was determined using the following formula: Acrosin activity $\left(\mu \mathrm{IU} / 10^{6}\right.$ spermatozoa $)=[$ sample optical density $(\mathrm{OD})$-control

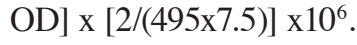

Colorimetric assay for seminal plasma zinc. The zinc concentrations in seminal fluid samples for each group were analyzed using the Seminal Plasma Zinc Quantitative assay kit (Huakang Biotechnology Development Co., Ltd.) according to the manufacturer's protocol. Seminal fluid $(1 \mathrm{ml})$ was centrifuged at $1,800 \times g$ for $10 \mathrm{~min}$ at $24^{\circ} \mathrm{C}$. The supernatant was subsequently transferred into a separate test tube for seminal plasma analysis. Physiological saline solution (1 ml) was used to wash the sediment, prior to the mixing of samples using a vortex-type mixer for $30 \mathrm{sec}$, and centrifugation for at $1,800 \mathrm{x} g$ for $10 \mathrm{~min}$ at $24^{\circ} \mathrm{C}$. The supernatant was removed, and the 
sediment was used to determine the zinc concentration instead of using $200 \mu \mathrm{l}$ of liquid sample. The absorbance of the sample solutions was read at $490 \mathrm{~nm}$. Sample zinc concentrations were calculated using the following formula: Seminal plasma zinc $(\mu \mathrm{mol})=$ zinc concentration $(\mathrm{mmol} / \mathrm{l}) \times$ semen volume $(\mathrm{ml})$.

Detection of sperm DNA integrity. DNA integrity analysis of sperm in fresh semen samples was performed using a the Sperm DNA Fragments Staining kit (Huakang Biotechnology Development Co., Ltd.), which is based on the emission of fluorescence signals from individual sperm stained with acridine orange (AO). The AO molecules intercalate into double-stranded DNA, and green fluorescence is emitted from the sperm nuclei. The DNA in sperm with immature nuclei can be denatured into single strands, which leads to the aggregation of $\mathrm{AO}$ molecules in the nuclei and emission of an orange-red fluorescence signal. The cell suspension was pipetted onto a glass slide and observed under a BX51 fluorescence microscope (Olympus Corporation, Tokyo, Japan) with a 480-490 nm filter. The percentage of green (normal DNA integrity) and orange-red (abnormal DNA integrity) spermatozoa/200 spermatozoa in each sample was determined by a single investigator. An abnormal sperm nuclear DNA integrity was considered to be when $>34 \%$ of sperm nuclei emitted orange-red fluorescence signals following AO staining.

Resveratrol treatment and dose preparation. The sperm suspensions from obese patients with astenospermia (60 cases) were pooled and divided randomly into the following three drug treatment groups: i) the control group, where spermatozoa were treated with Quinn's Advantage ${ }^{\mathrm{TM}}$ Fertilization (HTF) Medium (SAGE-In vitro Fertilization, Inc., Trumbull, CT, USA); ii) the negative control group, where spermatozoa were treated with Quinn's Advantage ${ }^{\mathrm{TM}}$ Fertilization (HTF) Medium plus $0.1 \%$ dimethyl sulfoxide (DMSO); and iii) the experimental group, which was subdivided into six subgroups based on the concentration of resveratrol $(2.6,6,15,30,50$, $100 \mu \mathrm{mol} / 1$; Sigma-Aldrich; Merck Millipore, Darmstadt, Germany) added to the medium, which was maintained at $37^{\circ} \mathrm{C}, 5 \% \mathrm{CO}_{2}$ and $95 \%$ humidity. Samples were incubated at $37^{\circ} \mathrm{C}$ for $30 \mathrm{~min}$ with resveratrol before sperm motility, seminal plasma zinc concentration and spermatozoa acrosin activity were analyzed.

Statistical analysis. Statistical analyses were performed using the SPSS software program (version 17.0; SPSS, Inc., Chicago, IL, USA). Normally distributed data were expressed as the mean \pm standard deviation. To verify the normality of the distribution, the Shapiro-Wilk test was performed, and one-way analysis of variance was used to compare the mean among the different groups. Variables with a non-normal distribution were analyzed using a Mann-Whitney U test or Kruskal-Wallis variance analysis test. $\mathrm{P}<0.05$ was considered to indicate a statistically significant difference.

\section{Results}

Distribution of male infertility frequency over the BMI groups. A total of 324 men (mean age, 37.2 years) were recruited to the study. Based on the analysis results of the semen parameters,
Table I. Distribution of male infertility frequency across the BMI groups.

\begin{tabular}{lccr}
\hline BMI group $^{\text {a }}$ & Fertile men (\%) & Infertile men (\%) & Total \\
\hline BMI $<18.5$ & $17(23.29)$ & $56(76.71)$ & 73 \\
$18.8 \leq \mathrm{BMI}<23$ & $50(60.98)$ & $32(39.02)$ & 82 \\
$23 \leq \mathrm{BMI}<25$ & $48(50.53)$ & $47(49.47)$ & 95 \\
$\mathrm{BMI} \geq 25$ & $24(32.43)$ & $50(67.57)$ & 74 \\
Total & $139(42.90)$ & $185(57.10)$ & 324
\end{tabular}

${ }^{\text {aS }}$ Subjects were divided into BMI groups according to the International Association for the Study of Obesity criteria (17). Male subjects included in the current study were referred to the Reproductive Medicine Center of Shanxi Women and Infants Hospital (Taiyuan, China) between January 2013 and January 2015, and were all of Han ethnicity. BMI, body mass index.

139 males $(42.90 \%)$ were classified as fertile and $185(57.10 \%)$ were classified as infertile. The distribution of male fertility in all BMI groups is shown in Table I. The general characteristics of the participants were stratified according to the four BMI groups, where 56/73 (76.71\%) of underweight males were infertile, $32 / 82(39.02 \%)$ of males with a normal weight were infertile, $47 / 95(49.47 \%)$ of overweight males were infertile and $50 / 74(67.57 \%)$ of obese males were infertile.

Comparison of routine semen parameters and serum sex hormone levels among BMI groups. As shown in Table II, routine semen parameters and serum sex hormone levels were assessed in abnormal weight groups, and the results were compared with those of the normal weight group. No significant difference in FSH, LH and PRL levels were observed among the abnormal weight groups and the normal weight group $(\mathrm{P}>0.05)$. In addition, the underweight group demonstrated no significant alterations in semen volume when compared with the normal weight group $(\mathrm{P}>0.05)$, whereas overweight and obese groups exhibited significantly lower semen volumes $(\mathrm{P}=0.0248$ and $\mathrm{P}=0.0142$, respectively). The percentage of sperm with progressive motility in the overweight group was not significantly different when compared with the normal weight group $(\mathrm{P}>0.05)$, whereas the percentage of sperm with progressive motility in the underweight and obese groups was significantly decreased ( $\mathrm{P}=0.0009$ and $\mathrm{P}=0.0419$ respectively). When compared with the normal weight group, the sperm concentration (underweight vs. normal weight, $\mathrm{P}<0.0001$; overweight vs. normal weight, $\mathrm{P}=0.0185$; obese vs. normal weight, $\mathrm{P}=0.0034)$, the percentage of sperm with a normal morphology (underweight vs. normal weight, $\mathrm{P}<0.0001$; overweight vs. normal weight, $\mathrm{P}=0.0396$; obese vs. normal weight, $\mathrm{P}=0.0004$ ) and the testosterone levels (underweight vs. normal weight, $\mathrm{P}=0.0011$; overweight vs. normal weight, $\mathrm{P}<0.0001$; obese vs. normal weight, $\mathrm{P}<0.0001$ ) in abnormal weight groups were significantly decreased. By contrast, E2 levels were significantly increased in underweight, overweight and obese groups when compared with the normal weight group $(\mathrm{P}=0.0003, \mathrm{P}<0.0001$ and $\mathrm{P}<0.0001$, respectively). 
Table II. Comparison of routine semen parameters and serum sex hormone levels among BMI groups.

\begin{tabular}{|c|c|c|c|c|}
\hline Parameter & $\begin{array}{c}\text { Normal weight } \\
(18.8 \leq \mathrm{BMI}<23)\end{array}$ & $\begin{array}{l}\text { Underweight } \\
(\mathrm{BMI}<18.5)\end{array}$ & $\begin{array}{c}\text { Overweight } \\
(23 \leq \mathrm{BMI}<25)\end{array}$ & $\begin{array}{c}\text { Obese } \\
(\mathrm{BMI} \geq 25)\end{array}$ \\
\hline Semen volume (ml) & $3.56 \pm 1.74$ & $3.54 \pm 1.68$ & $3.10 \pm 0.88^{\mathrm{a}}$ & $3.02 \pm 0.73^{\mathrm{a}}$ \\
\hline Sperm concentration $\left(\mathrm{x} 10^{6} / \mathrm{ml}\right)$ & $68.39 \pm 8.54$ & $59.42 \pm 8.16^{\mathrm{b}}$ & $65.39 \pm 8.22^{\mathrm{a}}$ & $64.39 \pm 8.19^{b}$ \\
\hline Progressive motility (\%) & $40.28 \pm 12.98$ & $33.62 \pm 11.31^{\mathrm{b}}$ & $39.56 \pm 11.74$ & $36.39 \pm 10.39^{\mathrm{a}}$ \\
\hline Morphology (\% normal) & $12.11 \pm 3.59$ & $7.63 \pm 1.33^{\mathrm{b}}$ & $11.08 \pm 3.32^{\mathrm{a}}$ & $10.21 \pm 2.9^{b}$ \\
\hline Follicle stimulating hormone (mIU/ml) & $6.98 \pm 2.55$ & $4.71 \pm 1.83$ & $5.11 \pm 2.24$ & $5.49 \pm 1.79$ \\
\hline Luteinizing hormone (mIU/ml) & $9.35 \pm 2.35$ & $9.1 \pm 1.32$ & $8.74 \pm 1.66$ & $8.63 \pm 1.29$ \\
\hline Estradiol (pg/ml) & $29.32 \pm 7.90$ & $34.11 \pm 8.27^{\mathrm{b}}$ & $36.63 \pm 7.53^{\mathrm{b}}$ & $37.21 \pm 8.94^{\mathrm{b}}$ \\
\hline Testosterone (ng/dl) & $386.58 \pm 21.32$ & $398.24 \pm 22.19^{b}$ & $369.76 \pm 19.38^{\mathrm{b}}$ & $354.71 \pm 19.23^{\mathrm{b}}$ \\
\hline Prolactin (mIU/ml) & $12.28 \pm 4.87$ & $12.26 \pm 3.48$ & $12.38 \pm 4.25$ & $12.45 \pm 4.71$ \\
\hline
\end{tabular}

${ }^{\mathrm{a}} \mathrm{P}<0.05$ and ${ }^{\mathrm{b}} \mathrm{P}<0.01$ vs. the normal weight group. BMI, body mass index.
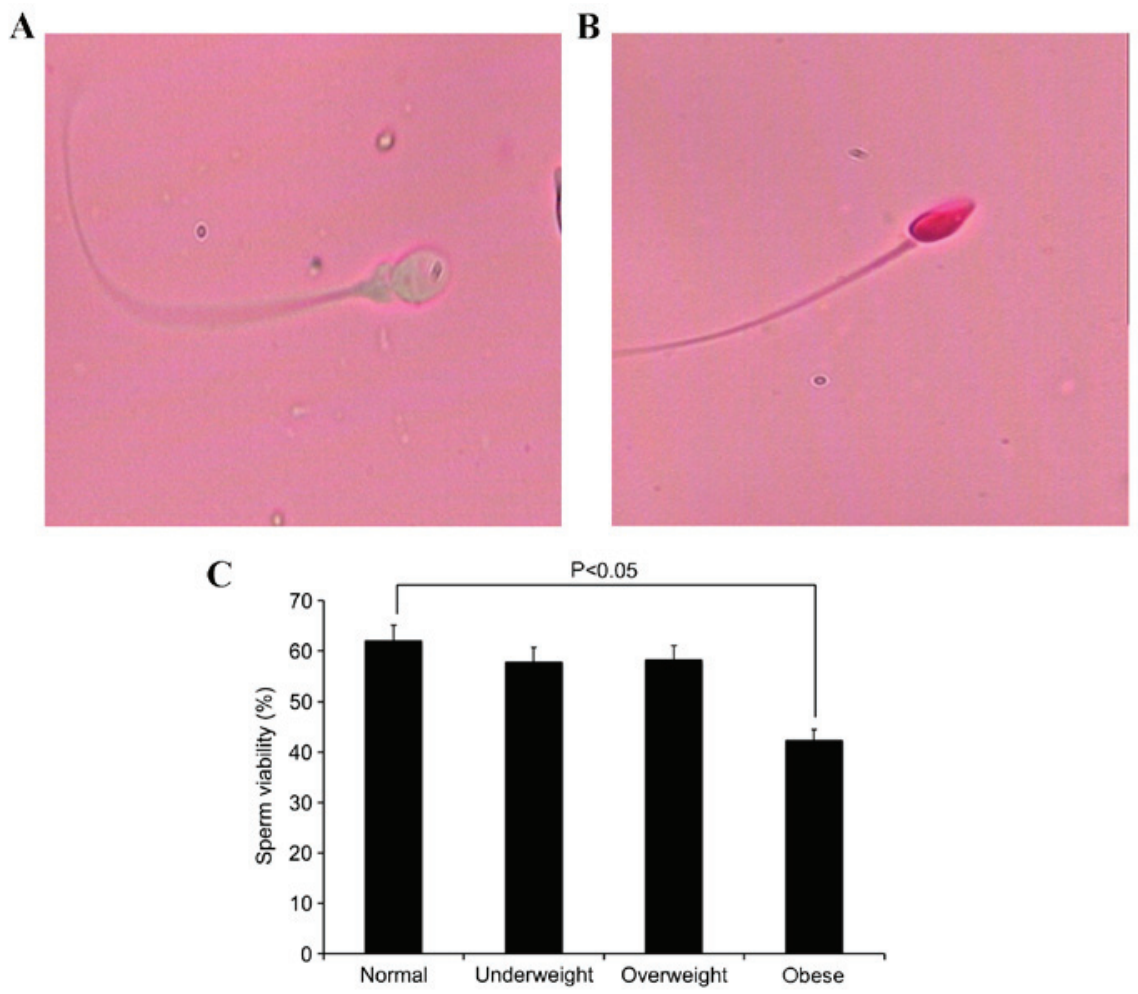

Figure 1. Comparison of sperm viability among BMI groups. Sperm viability was analyzed by eosin Y staining. Microscope images (magnification, x20) of (A) viable sperm (unstained sperm head) and (B) non-viable sperm (stained sperm head). (C) The obese group demonstrated a significant decrease in the number of viable sperm $(\mathrm{P}<0.05)$.

Comparison of sperm viability among BMI groups. Sperm viability was analyzed by eosin $\mathrm{Y}$ staining. The number of viable sperm (Fig. 1A) and non-viable sperm (Fig. 1B) in each group were examined using an Olympus CX31 microscope. When compared with the normal weight group, the obese group demonstrated a significant reduction in sperm viability $(\mathrm{P}=0.0297$; Fig. 1C).

Comparison of the plasma zinc concentration, spermatozoa acrosin activity and DNA fragmentation rates among BMI groups. As shown in Fig. 2A, the seminal plasma zinc concentration was significantly reduced in the obese group compared with the normal weight group $(\mathrm{P}=0.0233)$. Spermatozoa acrosin activity was analyzed using a UV spectrophotometric assay. Compared with normal weight group, the overweight and obese groups demonstrated a significant decrease in spermatozoa acrosin activity $(\mathrm{P}=0.0215$ and $\mathrm{P}=0.0193$, respectively; Fig. 2B). DNA fragmentation rates were analyzed by AO staining. As shown in Fig. 2C-E, spermatozoa emitting green (normal DNA integrity) and orange-red (abnormal DNA integrity) fluorescence signals were visualized and counted using an Olympus BX51 fluores- 
A

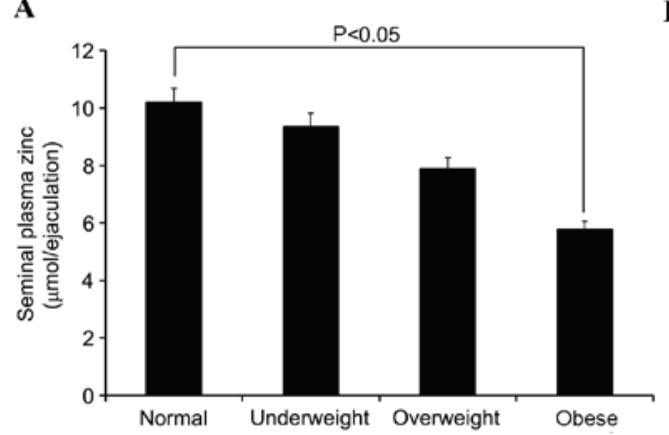

C

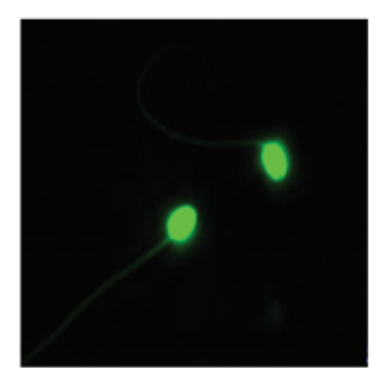

D

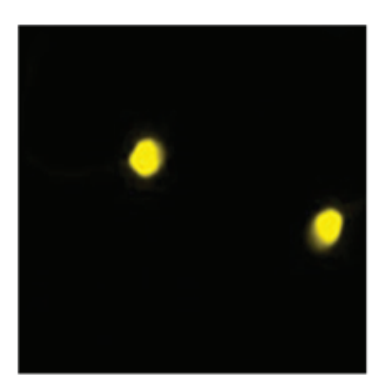

B

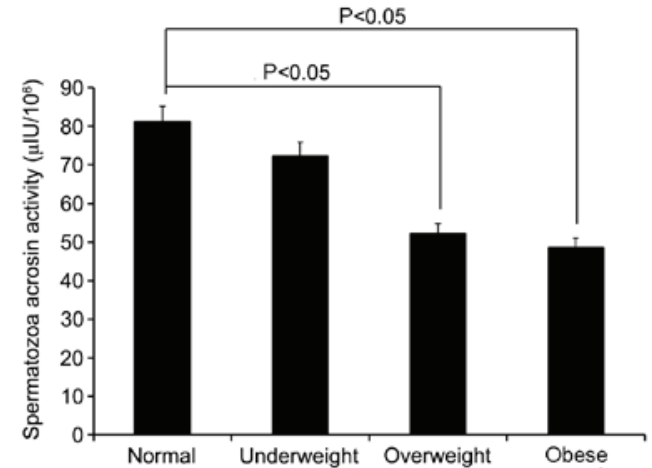

E

F

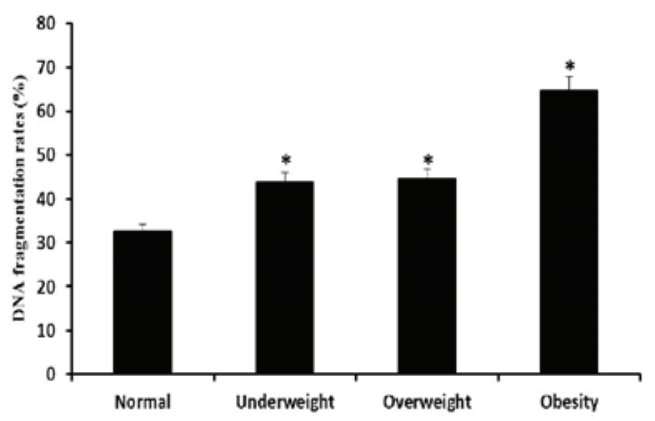

Figure 2. Plasma zinc concentration, spermatozoa acrosin activity and DNA fragmentation rates among BMI groups. (A) The seminal plasma zinc concentration was significantly reduced in the obese group compared with normal weight group $(\mathrm{P}=0.018)$. (B) The overweight and obese groups demonstrated a significant decrease in spermatozoa acrosin activity compared with the normal weight group ( $\mathrm{P}=0.031$ and $\mathrm{P}=0.021$, respectively). Representative fluorescence images of spermatozoa with (C) normal DNA integrity, and (D and E) abnormal DNA integrity are shown. DNA fragmentation rates were analyzed using acridine orange staining. (F) Spermatozoa from the underweight, overweight and obese groups demonstrated a significant increase in DNA fragmentation rates compared with those of the normal weight group $(\mathrm{P}=0.039, \mathrm{P}=0.042, \mathrm{P}=0.026$, respectively). " $\mathrm{P}<0.05$ vs. the normal weight group.

cence microscope with a 480-490 nm filter. When compared with the normal weight group, the underweight, overweight and obese groups demonstrated a significant increase in DNA fragmentation rates $(\mathrm{P}=0.0347, \mathrm{P}=0.0339$ and $\mathrm{P}=0.0208$ respectively; Fig. 2F).

Alterations in the progressive motility of sperm following treatment with increasing concentrations of resveratrol. As shown in Fig. 3, semen samples from obese patients with astenospermia (60 cases) treated with $0-100 \mu \mathrm{mol} / 1$ resveratrol for $30 \mathrm{~min}$, exhibited varying degrees of improvement in sperm motility. When semen samples were exposed to $30 \mu \mathrm{mol} / 1$ resveratrol, sperm motility was observed to increase compared with the other doses of resveratrol. Therefore, $30 \mu \mathrm{mol} / 1 \mathrm{resve}-$ ratrol was used for subsequent experiments.

Effect of resveratrol on plasma zinc concentration and spermatozoa acrosin activity. Semen samples were treated with quinn's

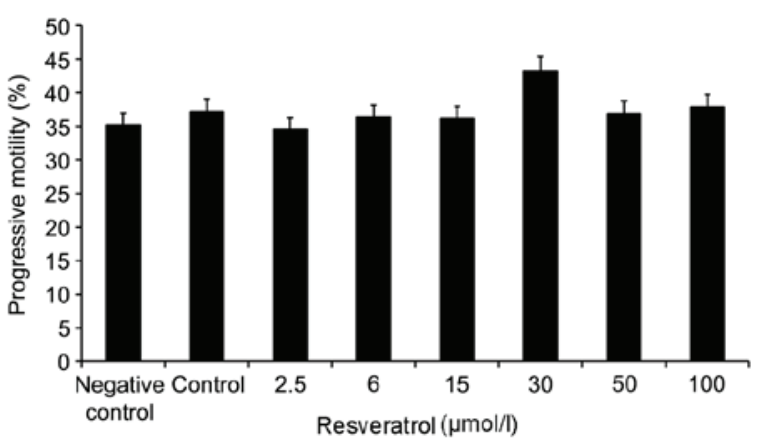

Figure 3. Alterations in the progressive motility of sperm treated with increasing concentrations of resveratrol. Semen samples from obese patients with astenospermia (60 cases) were treated with $0-100 \mu \mathrm{mol} / 1$ resveratrol for $30 \mathrm{~min}$, and sperm motility was altered to varying degrees. Exposure to $30 \mu \mathrm{mol} / 1$ resveratrol demonstrated the most notable improvement in progressive motility compared with control and negative control samples. The control and negative control groups were treated with Quinn's Advantage $\mathrm{e}^{\mathrm{TM}}$ Fertilization (HTF) Medium and Quinn's Advantage ${ }^{\mathrm{TM}}$ Fertilization (HTF) Medium plus $0.1 \%$ dimethyl sulfoxide, respectively. 
A

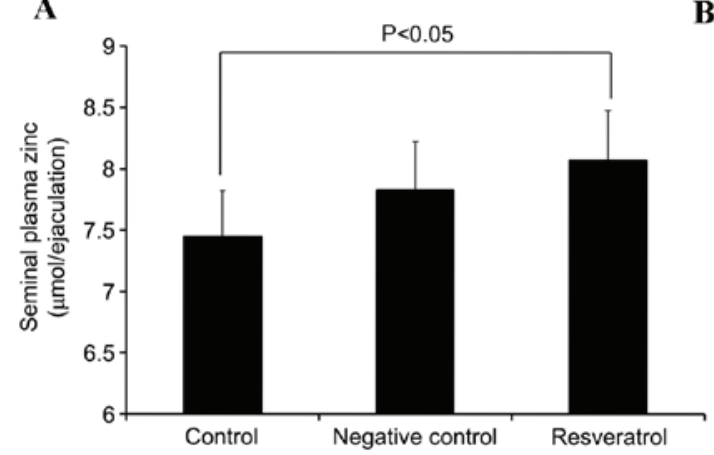

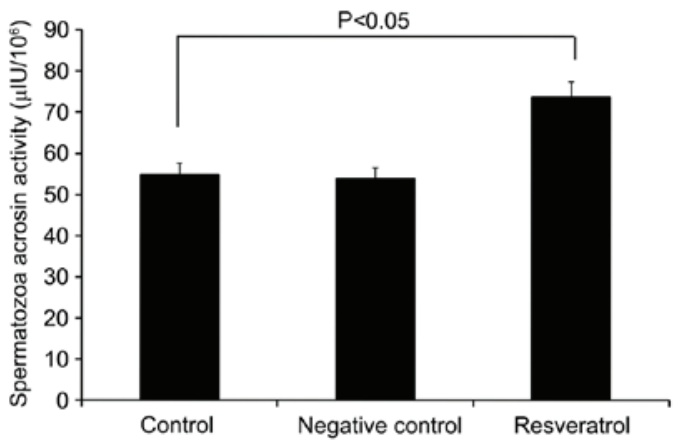

Figure 4. Effect of resveratrol on the plasma zinc concentration and spermatozoa acrosin activity. The resveratrol group exhibited a significant increase in (A) seminal plasma zinc concentrations and (B) spermatozoa acrosin activity when compared with the control group $(\mathrm{P}=0.023$ and $\mathrm{P}=0.031$, respectively).

1020 nutrient solution (control), quinn's 1020 nutrient solution plus $0.1 \%$ DMSO (negative control) or $30 \mu \mathrm{mol} / 1$ resveratrol for $30 \mathrm{~min}$, and the plasma zinc concentration and spermatozoa acrosin activity was analyzed. As shown in Fig. 4A, the seminal plasma zinc concentration increased significantly in the resveratrol group compared with the control group $(\mathrm{P}=0.0199)$. In addition, the resveratrol group demonstrated a significant increase in spermatozoa acrosin activity when compared with the control group ( $\mathrm{P}=0.0248$; Fig. 4B).

\section{Discussion}

Excess body weight and obesity, defined by the WHO as the abnormal or excessive accumulation of fat, is posing a growing threat to health in countries worldwide $(20,21)$. Excessive weight is not only linked to an increased risk of developing chronic disease, but has also been demonstrated to increase the risk of developing reproductive disorders $(8,22,23)$. The association between excessive weight and infertility has been established in females, but not in males. Females who are overweight or obese are more likely to experience ovulatory and menstrual disorders, and may experience delayed childbearing as a consequence (8). Several studies have investigated the association between BMI and different sperm parameters (24). There is evidence to suggest that sperm concentration and total motile sperm count are detrimentally affected by a high BMI (23-25). In addition, previous studies have demonstrated that low $\left(<18.5 \mathrm{~kg} / \mathrm{m}^{2}\right)$ and high $\left(\geq 25 \mathrm{~kg} / \mathrm{m}^{2}\right)$ BMIs are associated with reduced testicular volume and reduced semen quality, which suggests an impairment of spermatogenesis $(24,25)$. Similarly to females, a sex hormone imbalance may influence reproduction in men, and excessive weight may affect male hormone levels $(24,25)$. It has been reported that obesity is closely associated with endocrine disorders, such as sex hormone abnormalities $(26,27)$. It has been hypothesized that extended periods of obesity may induce alterations in the secretion and/or metabolism of hormones, leading to altered hormone-mediated effects on target organs (28). These associations are well-documented effects of excess body weight on the production and function of sex hormones (29). Thus, it is necessary to evaluate the effects of excess body weight and obesity on male infertility.

In the present study, males in the excess weight and obese groups demonstrated a significant decrease in semen volume compared with those of the normal weight group, whereas males in the underweight group did not exhibit any significant alterations. The progressive motility of sperm in the underweight and obesity groups was significantly reduced compared with the normal weight group, whereas no significant alteration was observed in the overweight group. When compared with the normal weight group, the sperm concentration, normal sperm morphology and testosterone levels in the abnormal weight groups demonstrated a significant decrease, whereas the E2 levels were significantly increased. No significant difference in FSH, LH and PRL levels among the abnormal weight groups and the normal weight group were observed. The seminal plasma zinc concentration was significantly reduced in the obese group compared with normal weight group. Spermatozoa acrosin activity was analyzed using a UV spectrophotometric assay. Compared with normal weight group, the overweight and obese groups demonstrated a significant decrease in spermatozoa acrosin activity. When compared with the normal weight group, the underweight, overweight and obese groups exhibited a significant increase in DNA fragmentation rates. These results are consistent with those reported in previous studies (6-8).

Taking into account that obesity may lead to a reduction in sperm quality, the aim of the present study was to determine whether resveratrol may improve the sperm quality in obese patients. Resveratrol (trans-3,5,4'-trihydroxystilbene), a phytoalexin that functions to protect plants from parasites or environmental stress (30), is a polyphenolic compound present in grapes, peanuts, berries and wine. A number of studies have reported that resveratrol possesses a wide variety of health-promoting properties, including anticancer, anti-inflammatory, antimicrobial, cardioprotective and antioxidant properties. Recent studies involving animal models have reported that resveratrol demonstrates therapeutic and protective effects against testicular damage, abnormal hormonal levels and semen parameters (11-13).

In the present study, following the treatment of semen samples from obese patients with astenospermia with $0-100 \mu \mathrm{mol} / 1$ resveratrol for $30 \mathrm{~min}$, sperm motility was improved to varying degrees. Among these concentrations of resveratrol, treatment with $30 \mu \mathrm{mol} / \mathrm{l}$ was associated with the most significant improvement in sperm motility when compared with controls. In addition, the seminal plasma zinc concentration increased significantly in resveratrol-treated group compared with control group. Furthermore, the resveratrol group demonstrated 
a significant increase in spermatozoa acrosin activity when compared with control and negative control groups. These data suggest that male obesity negatively impacts on fertility by directly altering sperm function and the molecular composition. Therefore, resveratrol may demonstrate therapeutic and protective effects against obesity-induced abnormal semen parameters. The results of the present study have not revealed the precise mechanisms underlying these protective effects; however, it is possible that the combined antioxidant and anti-apoptotic properties of resveratrol may serve an important role. Further studies are required to examine the molecular pathways responsible for the mechanisms underlying the effects of resveratrol on improving sperm motility, acrosin activity and plasma zinc concentrations.

\section{Acknowledgements}

The present study was supported by the Research Fund of National Health and Family Planning Commission of China (grant no. RFNHFPCC, 201402004).

\section{References}

1. Drobnis EZ and Johnson M: The question of sperm DNA fragmentation testing in the male infertility work-up: A response to Professor Lewis' commentary. Reprod Biomed Online 31: 138-139, 2015.

2. Whitfield M, Pollet-Villard X, Levy R, Drevet JR and Saez F: Posttesticular sperm maturation, infertility, and hypercholesterolemia. Asian J Androl 17: 742-748, 2015.

3. Hammoud AO, Wilde N, Gibson M, Parks A, Carell DT and Meikle AW: Male obesity and alteration in sperm parameters. Fertil Steril 90: 2222-2225, 2008.

4. Ring JD, Lwin AA and Köhler TS: Current medical management of endocrine-related male infertility. Asian J Androl 18: 357-363, 2016

5. Cissen M, Bensdorp A, Cohlen BJ, Repping S, de Bruin JP and van Wely M: Assisted reproductive technologies for male subfertility. Cochrane Database Syst Rev 2: CD000360, 2016.

6. Dağ ZÖ and Dilbaz B: Impact of obesity on infertility in women. J Turk Ger Gynecol Assoc 16: 111-117, 2015.

7. Katib A: Mechanisms linking obesity to male infertility. Cent European J Urol 68: 79-85, 2015.

8. Esmaeilzadeh S, Andarieh MG, Ghadimi R and Delavar MA: Body mass index and gonadotropin hormones (LH \& FSH) associate with clinical symptoms among women with polycystic ovary syndrome. Glob J Health Sci 7: 101-106, 2014.

9. Zhou Y, Chen K, He L, Xia Y, Dai W, Wang F, Li J, Li S, Liu T, Zheng Y, et al: The protective effect of resveratrol on concanavalin-A-Induced acute hepatic injury in mice. Gastroenterol Res Pract 2015: 506390, 2015

10. Lang F, Qin Z, Li F, Zhang H, Fang Z and Hao E: Apoptotic cell death induced by resveratrol is partially mediated by the autophagy pathway in human ovarian cancer cells. PLoS One 10: e0129196, 2015.

11. Orihuela-Campos RC, Tamaki N, Mukai R, Fukui M, Miki K, Terao J and Ito HO: Biological impacts of resveratrol, quercetin, and $\mathrm{N}$-acetylcysteine on oxidative stress in human gingival fibroblasts. J Clin Biochem Nutr 56: 220-227, 2015.

12. Jiao Y, Li H, Liu Y, Guo A, Xu X, Qu X, Wang S, Zhao J, Li Y and Cao Y: Resveratrol inhibits the invasion of Glioblastoma-Initiating cells via Down-Regulation of the PI3K/Akt/NF-kappaB signaling pathway. Nutrients 7: 4383-4402, 2015.
13. Liu J, Yi L, Xiang Z, Zhong J, Zhang H and Sun T: Resveratrol attenuates spinal cord injury-induced inflammatory damage in rat lungs. Int J Clin Exp Pathol 8: 1237-1246, 2015.

14. Sharma P, Huq AU and Singh R: Cypermethrin-induced reproductive toxicity in the rat is prevented by resveratrol. J Hum Reprod Sci 7: 99-106, 2014.

15. Eleawa SM, Alkhateeb MA, Alhashem FH, Bin-Jaliah I, Sakr HF, Elrefaey HM, Elkarib AO, Alessa RM, Haidara MA, Shatoor AS and Khalil MA: Resveratrol reverses cadmium chloride-induced testicular damage and subfertility by downregulating p53 and Bax and upregulating gonadotropins and $\mathrm{Bcl}-2$ gene expression. J Reprod Dev 60: 115-127, 2014.

16. Juan ME, González-Pons E, Munuera T, Ballester J, Rodríguez-Gil JE and Planas JM: trans-Resveratrol, a natural antioxidant from grapes, increases sperm output in healthy rats. J Nutr 135: 757-760, 2005.

17. Asia-Pacific Perspective: Redefining obesity and its treatment. Health Communications Australia: Melbourne 2000.

18. World Health Organization. WHO laboratory manual for the examination and processing of human semen, 5 th edn. Geneva: World Health Organization, 2010.

19. Auger J, Eustache F, Andersen AG, Irvine DS, Jørgensen N, Skakkebaek NE, Suominen J, Toppari J, Vierula M and Jouannet P: Sperm morphological defects related to environment, lifestyle and medical history of 1001 male partners of pregnant women from four European cities. Hum Reprod 16: 2710-2717, 2001.

20. McTigue KM, Hess R and Ziouras J: Diagnosis and treatment of obesity in the elderly. AHRQ Technology Assessments. Rockville (MD), 2003.

21. Goulão B, Santos O and Carmo Id: The impact of migration on body weight: A review. Cad Saude Publica 31: 229-245, 2015

22. Jungheim ES, Schon SB, Schulte MB, DeUgarte DA, Fowler SA and Tuuli MG: IVF outcomes in obese donor oocyte recipients: A systematic review and meta-analysis. Hum Reprod 28: 2720-2727, 2013

23. Sermondade N, Faure C, Fezeu L, Shayeb AG, Bonde JP, Jensen TK, Van Wely M, Cao J, Martini AC, Eskandar M, et al: BMI in relation to sperm count: An updated systematic review and collaborative meta-analysis. Hum Reprod Update 19: 221-231, 2013.

24. Samavat J, Natali I, Degl'Innocenti S, Filimberti E, Cantini G, Di Franco A, Danza G, Seghieri G, Lucchese M, Baldi E, et al: Acrosome reaction is impaired in spermatozoa of obese men: A preliminary study. Fertil Steril 102: 1274-1281.e2, 2014.

25. Macdonald AA, Stewart AW and Farquhar CM: Body mass index in relation to semen quality and reproductive hormones in New Zealand men: A cross-sectional study in fertility clinics. Hum Reprod 28: 3178-3187, 2013.

26. Hammoud AO, Meikle AW, Peterson CM, Stanford J, Gibson M and Carrell DT: Association of 25-hydroxy-vitamin D levels with semen and hormonal parameters. Asian J Androl 14: 855-859, 2012.

27. Eskandar M, Al-Asmari M, Babu Chaduvula S, Al-Shahrani M, Al-Sunaidi M, Almushait M, Donia O and Al-Fifi S: Impact of male obesity on semen quality and serum sex hormones. Adv Urol 2012: 407601, 2012.

28. Yan WJ, Mu Y, Yu N, Yi TL, Zhang Y, Pang XL, Cheng D and Yang J: Protective effects of metformin on reproductive function in obese male rats induced by high-fat diet. J Assist Reprod Genet 32: 1097-1104, 2015.

29. Hofny ER, Ali ME, Abdel-Hafez HZ, Kamal Eel-D, Mohamed EE, Abd El-Azeem HG and Mostafa T: Semen parameters and hormonal profile in obese fertile and infertile males. Fertil Steril 94: 581-584, 2010.

30. Trotta V, Lee WH, Loo CY, Haghi M, Young PM, Scalia S and Traini D: In vitro biological activity of resveratrol using a novel inhalable resveratrol spray-dried formulation. Int J Pharm 491: 190-197, 2015. 